\title{
Control of a Rigid-Flexible Satellite Solar Panel with Non-collocated SensorlActuator
}

\author{
Luiz Carlos Gadelha de Souza ${ }^{1,2}$ \\ 1. Aerospace Department, University of Brasilia, Brasilia 72444-240, Brazil \\ 2. Space Mechanics and Control Division, National Institute for Space Researcher, São José dos Campos 12227-010, Brazil
}

Received: December 25, 2014 / Accepted: January 14, 2015 / Published: March 25, 2015.

\begin{abstract}
This paper presents a model and analysis for a flexible link with non-collocations of sensors and actuators. It shows the changes in the system dynamics and the appearance of zeroes in the right-plan complex, turning the system a non-minimum phase system. The performance of the PID (proportional-integral-derivative) and LQR (linear quadratic regulator) controller are discussed considering the zero dynamics of the system in three points of special interest: (1) the collocated case, when the sensor is in the base of the link; (2) the critical case, where the system starts to present zeroes in the right-plan complex and (3) the limit case, when the sensors are in the end point of the flexible link. Investigation for a simple rigid-flexible model with one mode, in the three cases, the PID and LQR controller performance are damage. To deal with this kind of problem, new control techniques should be developed.
\end{abstract}

Key words: Satellite control, flexible system, non-collocated sensor and actuator.

\section{Introduction}

The artificial satellites are space structures composed by a central body with actuators, sensors, equipment's and flexible links as the solar panels. Two important construction parameter that directly influences the system dynamics are the panel flexibility and the distance between the attitude sensors and actuators which are collocated over the satellite body. Sensors and actuators are known as non-collocation system while they are not located at the same place $[1,2]$. The non-collocation of sensors/actuators has great influence in the satellite ACS (attitude control system) performance, since it can induce zeroes on the right complex plan, configuring a non-minimum phase system. Wang [3] presented the effect of non-collocation of sensors and actuators on flexible structures and concluded that LQR (linear quadratic regulator) is not sufficient to stabilize the system, being necessary to project a

Corresponding author: Luiz Carlos Gadelha de Souza, Ph.D., research field: satellite control system. E-mail: lcgs@unb.br.
LQG/LTR (linear quadratic gaussian/loop transfer recovery). Many others authors discussed the theme and design other controllers for this problem. A different approach, also based on an optimization of weight matrix was applied in Refs. [4, 5] to design a control system of flexible satellites that has presented sensor/actuator location problem. Another problem related to stability of rigid-flexible satellite is associated with fuel tanks inside the satellite when it is subject to large angle manoeuvre [6]. The flexibility motion and/or liquid vibration can introduce a tracking error resulting in a minimum attitude acquisition time [7]. A detailed investigation of the influence of the panel's flexibility into the ACS design has been done by Ref. [8]. An experimental controller robustness investigation associated with parameters variation was done by Ref. [9], where the estimation of the platform inertia parameters was introduced as part of the platform ACS design. The problem of designing satellite non-linear controller for rigid satellite has been done by Ref. [9] using the SDRE (state dependent Riccati equation) method 
which is able to deal with high non-linear plants. Due to the complexity of modelling the fluid and/or flexible dynamic of the system, it is common to use mechanical systems analogies that describe this dynamic. Besides, if one needs to know some physical parameters related with the slosh or the flexibility dynamics, it is common to obtain then by experimental apparatus or some kind of estimating method such as Kalman filter developed a control law to improve performance to drive rotary system. The optimal placement of the sensors/actuators of these systems analysed by Ref. [10] has projected H-infinity controller for non-collocated flexible structures. An extension of that concept with optimal sensor/actuator placement using a modified H-infinity control technique with a genetic algorithm was applied by Ref. [11]. This paper presents a preliminary investigation about the sensor/actuator location problem. One considers a simple rigid-flexible satellite and analyse the behaviours of the zeroes when the system chance from minimum phase to non-minimum phase situation. In the sequel, one design a PID (proportional-integral-derivative) and LQR controllers in order to show that traditional control techniques can have its performance damage when the system are non-minimum phase. These result, indicates that new control techniques need to be investigate to improve control system performance. The paper is organized as follows: Section 2 formulates the mathematical model using the Lagrangian formalism; Section 3 performs the zero dynamics analysis of the system; Section 4 investigates the design and the performance of the PID and LQR controllers; Section 5 gives conclusions.

\section{Mathematical Model}

The mathematical model used in this paper was formulated using the Lagrangian formalism. The model adopted is a pseudo-pinned model, in that the base of the link is considered pinned and the link can vibrate in one axis. There is a concentrated mass $M_{p}$ in the point of link with inertia $J_{p}$.

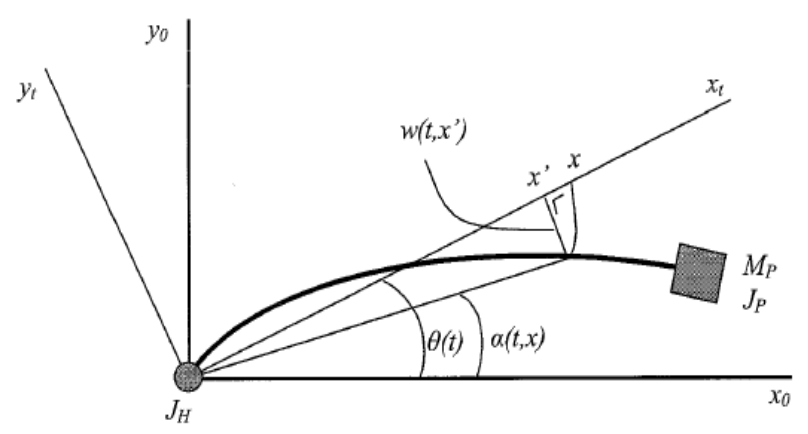

Fig. 1 Flexible parameters and coordinates system.

The kinetic energy of the all structure is given by Eq. (1):

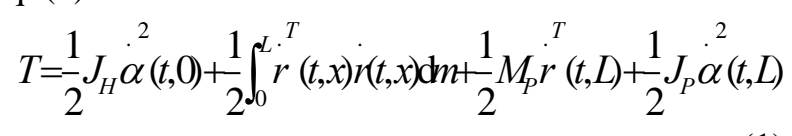

where, $T$ is the kinetic energy, $J_{H}$ is the hub inertia, $\alpha$ is the vibration angle, $r$ is the vector position, $M_{p}$ is the concentrated mass in the end of the hub, $J_{p}$ is the inertia associated with the mass $M_{p}, L$ is the length of the flexible structure.

The potential energy is given by Eq. (2):

$$
V=\frac{1}{2} \int_{0}^{L}\left[\frac{\partial^{2} \omega}{\partial x^{2}}\right]^{2} \mathrm{~d} x
$$

The derivation of equations of motion has been done using the Lagrangian formalism. The elastic displacement can be written as a function of the time and space.

$$
\omega_{p}(t, x)=q_{p}(t) \Phi_{p}(x)
$$

Substituting the previous equations in the Lagrange equations and after some manipulation the equation of motion is given by Eqs. (4) and (5).

$$
\begin{gathered}
E I \Phi_{p}^{[4]}(x)+\rho \omega^{2}\left(\Phi_{p}(x)+\frac{x}{J} v_{0}\right)=0 \\
q_{p}(t)+\omega^{2} q_{p}(t)=0
\end{gathered}
$$

The initial conditions for the problem are

$$
\begin{aligned}
& \Phi_{p}(0)=0 \\
& E I \Phi_{p}^{\prime \prime}(0)=-\omega^{2} J_{H} \Phi_{p}^{\prime}(0) \\
& E I \Phi_{p}^{\prime \prime}(L)=\omega^{2} J_{P} \Phi_{p}^{\prime}(L) \\
& E I \Phi_{p}^{\prime \prime}(L)=-\omega^{2} M_{P} \Phi_{p}^{\prime}(L)
\end{aligned}
$$


The general solution of the system is:

$$
\begin{gathered}
\Phi p(x)=A \sin (\beta x)+B \cos (\beta x)+C \sinh (\beta x) \\
+D \cosh (\beta x)+F \\
q(t)=q(0) \cos (\omega t)+q(0) \sin (\omega t)
\end{gathered}
$$

Assuming that $B=-D$ is possible to simplify to obtain the natural frequencies given by Eq. (9):

$$
\omega_{i}=\beta_{i}^{2} \sqrt{\frac{E I}{\rho}}, i=1,2 . .
$$

These equations in the linear model form is:

$$
M_{p} \ddot{q}_{p}+K_{p} q_{p}=F_{p} \tau
$$

where,

$$
M_{p}=\left(\begin{array}{cc}
J & 0^{T} \\
0 & I
\end{array}\right), K_{p}=\left(\begin{array}{ll}
0 & 0^{T} \\
0 & \omega^{2}
\end{array}\right), F_{p}=\left(\begin{array}{l}
1 \\
\Phi^{\prime}(0)
\end{array}\right)
$$

In the space state form, one has:

$$
\begin{aligned}
& \dot{x}=A x+B u \\
& x=\left[\begin{array}{ll}
q_{p} & \dot{q}_{p}
\end{array}\right]^{T} \\
& A=\left(\begin{array}{cc}
0 & I \\
-M_{p}^{-1} K_{p} & 0
\end{array}\right) \\
& B=\left(\begin{array}{l}
0 \\
-M_{p}^{-1} F_{p}
\end{array}\right) \\
& u=\tau
\end{aligned}
$$

The state $x$ represents the elastic displacement and its variation with time and $u$ is the control law to be designed. If damping is considered in the model the matrices $A$ and $D$ are modified becoming:

$$
\begin{aligned}
& A=\left(\begin{array}{cc}
0 & I \\
-M_{p}^{-1} K_{p} & -M_{p}^{-1} D_{p}
\end{array}\right) \\
& D_{p}=\left(\begin{array}{cc}
b_{H} & 0^{T} \\
0 & 2 \omega \psi
\end{array}\right)
\end{aligned}
$$

\section{Zero Dynamics Analysis}

In the zero dynamics analysis, three points have specially interest:

(1) the collocated case, when the sensor is in the base of the link $(x=0)$;
(2) the critical case, where the system starts to present zeroes in the right-plan complex $(x=0.7888$ L);

(3) the limit case, when the sensors are in the end point of the flexible link $(x=L)$.

The zero dynamics are also related with the damping of the system, besides the position of the sensors along the structure.

The parameters data used in the model are: $L=$ $1,000 \mathrm{~m}, h=50 \mathrm{~mm}, b=3 \mathrm{~mm}, \rho=4 \mathrm{~kg} / \mathrm{m}^{3}, I=1.2$ $\mathrm{Kg} \mathrm{m}^{2}$. It is considered $J_{h}=0.01, J_{p}=0, M_{p}=0, \xi=$ $0.01, b_{h}=0.05$.

It can be observed in Figs. 1 and 2 that in the cases that the system is considered collocated and with small damping in the system, the Bode diagram do not present overshoots beyond resonance in natural frequencies and all the poles and zeroes stay in the left-plan complex.

If the position of the sensor varies along the structure, it is possible to find the position where the system start to presented zeroes in the right-plan complex, in other words, it becomes to be a non-minimum phase system. For the structure considered, the transition point is located in $78.88 \%$ of the length, starting in the base of the flexible link. In this frequency, an anti-resonant frequency appears as illustrated in the previously Bode diagram of Fig. 4 and the zeroes cross the imaginary axis of Fig. 5.

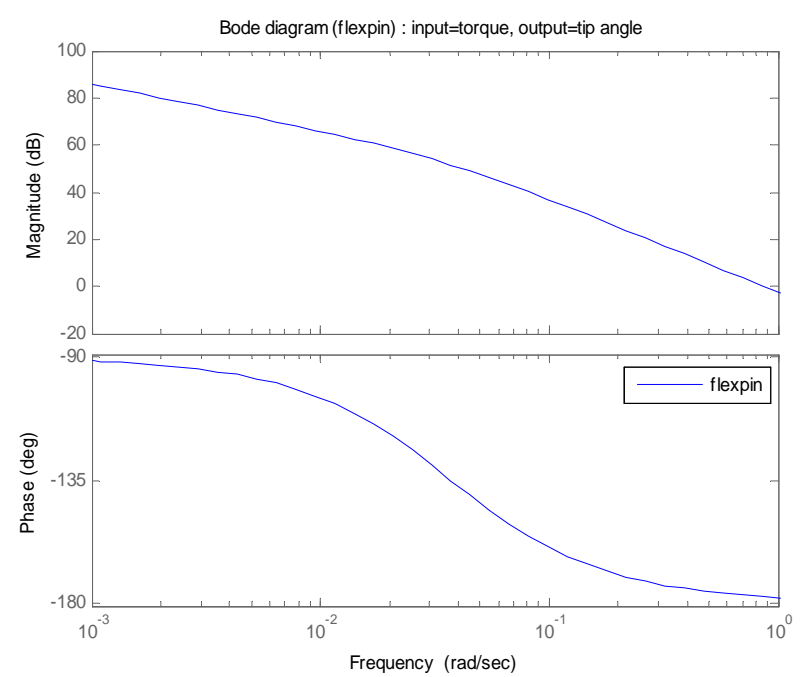

Fig. 2 Bode diagram sensor located at $x=0$. 


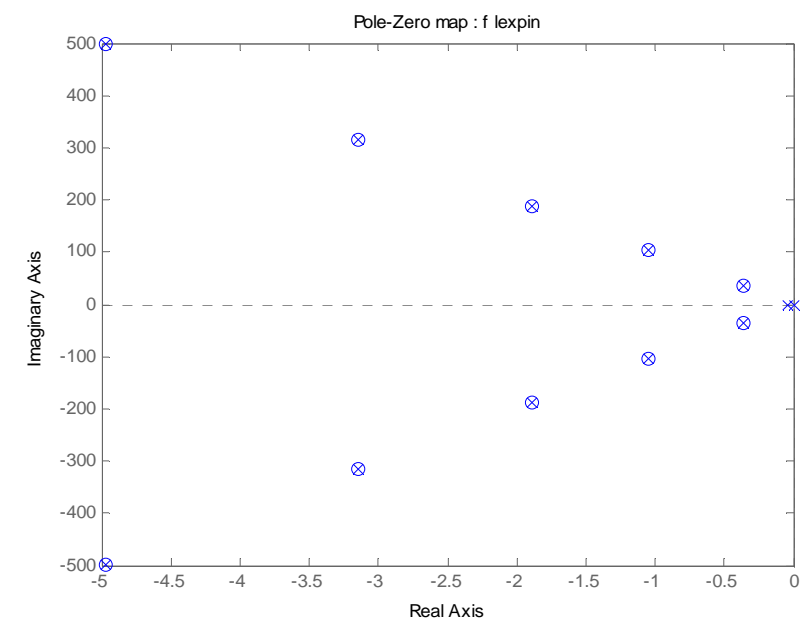

Fig. 3 Pole-Zero map sensor located at $x=0$.

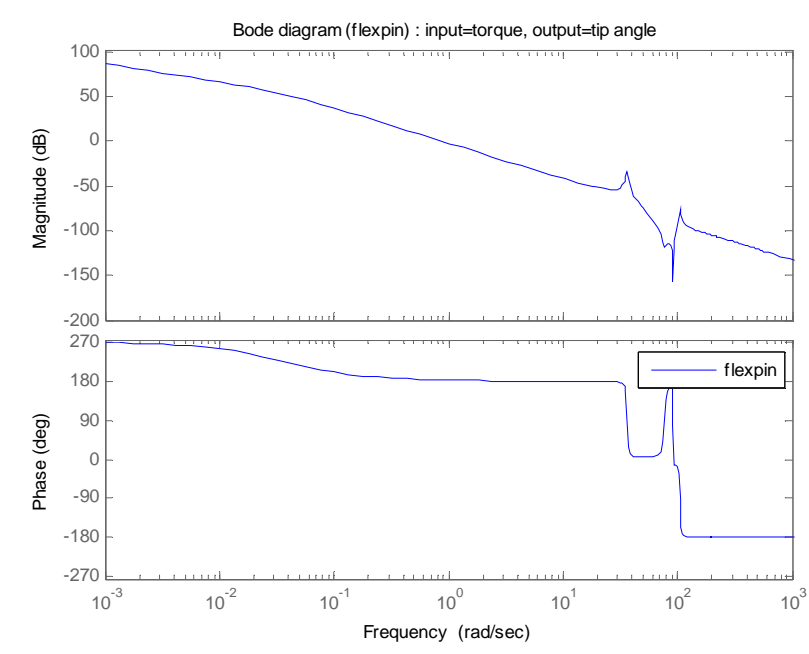

Fig. 4 Bode diagram sensor located at $x=0.788 \mathrm{~L}$.

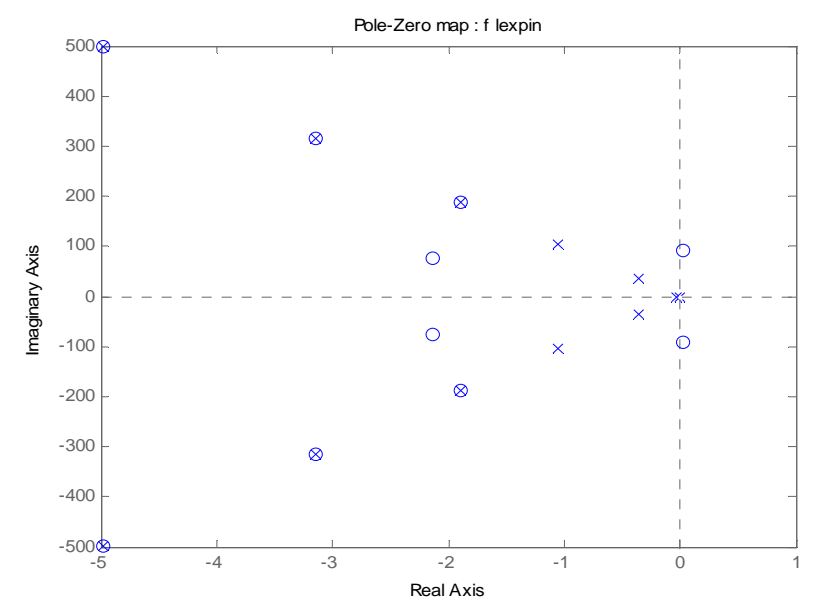

Fig. 5 Pole-Zero map sensor located at $x=0.7888 \mathrm{~L}$.

Finally, Figs. 6 and 7 shows the limit case, when the sensors are in the end point of the flexible link $(x=$
$L$ ), which characterizes the non-collocated sensor/actuator with small damping in the system. One observes, that the Bode diagram present overshoots just in the resonance natural frequencies and that some the zeroes move and stay in the right-plan complex.

From the investigation, one has observed that the zero dynamics of the system is too sensitive to the sensor/actuator location and damping. This is a very important point when designing the ACS for rigid-flexible satellite because the sensor/actuation problem can degrade the accuracy of the satellite pointing. And it will be shown in the next section.

The transfer functions for the three cases are showed in Table 1, from which one can observe that the poles of the system do not chance. But, the great change of the zeroes position happens from the sensor position $x=0.7881$ to $x=L$, due to the change in the numerator signal of the transfer function.

\section{Controller Design}

The controller design is performed based on the linear space state system model, considering that the system has only one flexible mode. The LQR controller is designed, based on the optimal control theory [6] which objective is to minimize the functional given by Eq. (12):

$$
J_{L Q R}=\int_{0}^{\infty}\|z(t)\|^{2}+\rho\|u(t)\|^{2} \mathrm{~d} t
$$

where, $z$ are the states, rho is the design tuning parameters and $u$ is the control law is given by

$$
u=-K x
$$

where, the gain $K$ is given by Eq. (14):

$$
K=\left(H^{\prime} Q H+\rho R\right)^{-1}\left(B^{\prime} P+H^{\prime} Q G\right)
$$

which is associated with the solution of the algebraic Riccati equation given by Eq. (15):

$$
\begin{aligned}
& A^{T} P+P A+G^{T} Q G-\left(P B+G^{T} Q H\right) * \\
& \left.H^{T} Q H+\rho R\right)^{-1}\left(B^{T} P+H Q G\right)=0
\end{aligned}
$$

Let us consider as a performance specification that the controller must follow a reference position of $30^{\circ}$ 
Table 1 Transfer functions.

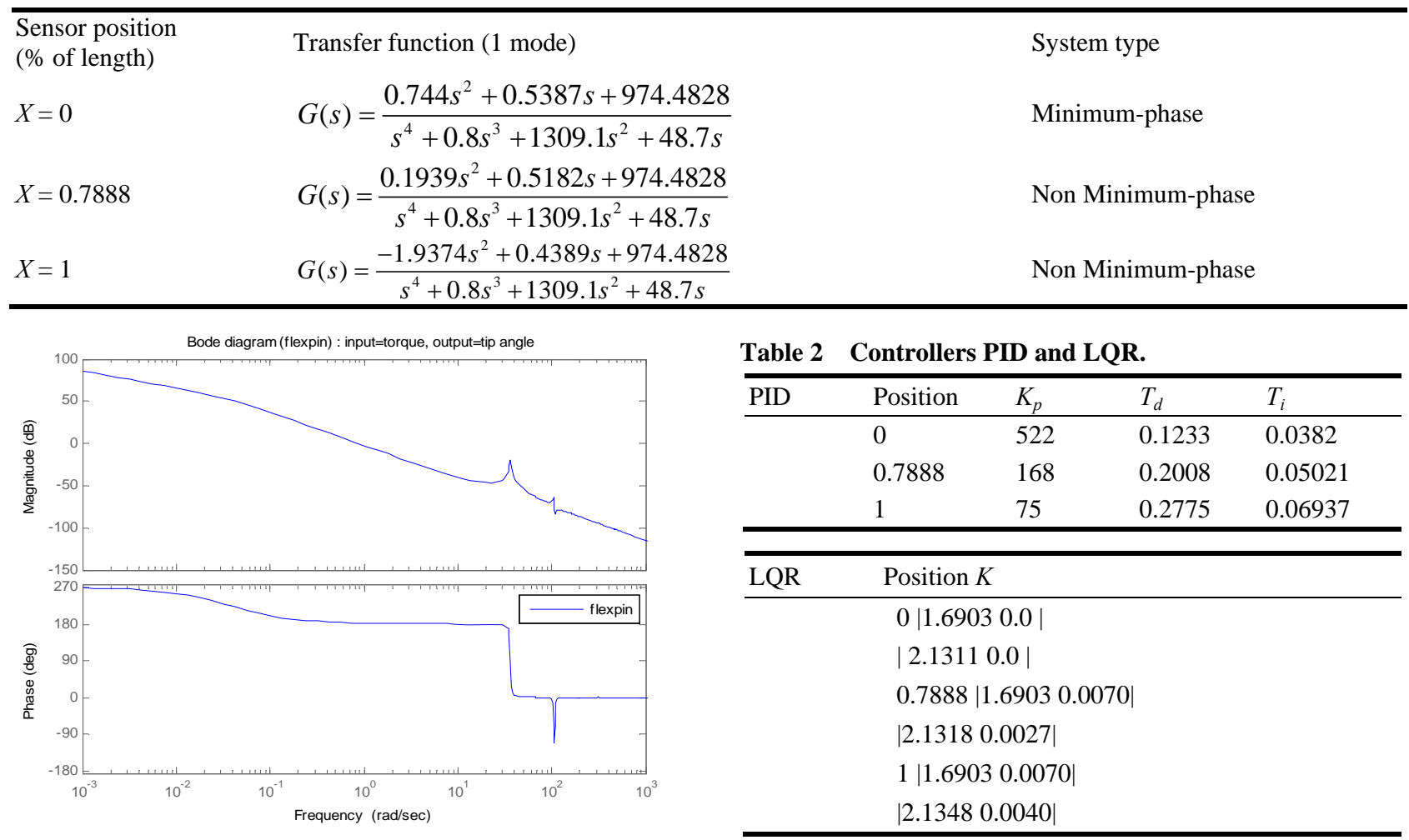

Fig. 6 Bode diagram sensor located in $x=L$.

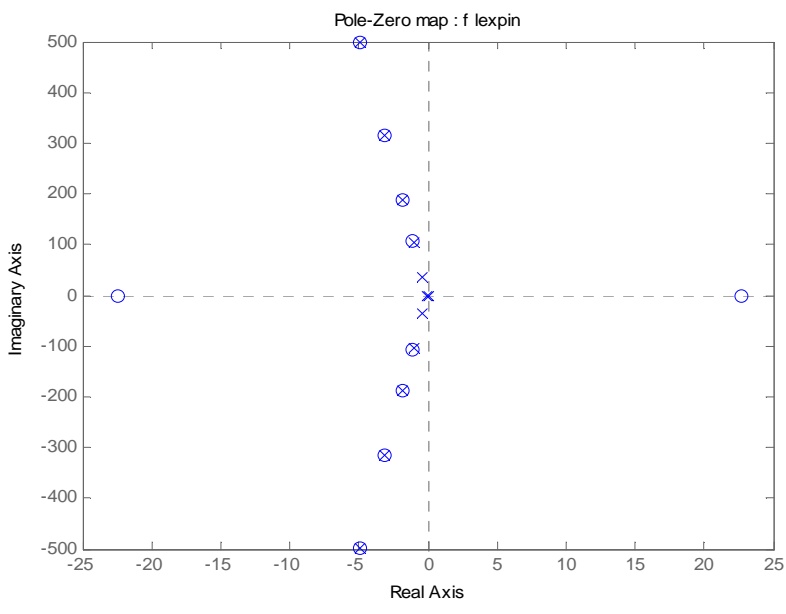

Fig. 7 Pole-Zero map sensor located in $x=L$.

and that the initial condition of satellite position and velocity are zero. In order to compare the LQR controller performance, one has also designed a traditional PID controller. The PID gains $K_{p}, T_{i}$ and $T_{d}$ and the LQR gains are showed in Table 2.

The PID and LQR controllers performance are showed in Figs. 8-10 for the cases that the sensor is located in $x=0, x=0.788 \mathrm{l}$ and $x=L$, respectively.

\section{Conclusions}

This paper presents a preliminary investigation about the sensor/actuator location problem. One considers a simple rigid-flexible satellite and analyse the behaviours of the zeroes when the system chance from minimum phase to non-minimum phase situation. In the sequel, one design a PID and LQR controllers in order to show that traditional control techniques can have its performance damage when the system are non-minimum phase. These result, indicates that new control techniques need to be investigate to improve control system performance. Besides, from that investigation, considering that simple rigid-flexible link model, one can observe that the non-collocated sensor/actuator problem is very sensitive and that the sensor wrong position can result in the appearance of zeroes in the right half-plane. Therefore, when rigid-flexible satellite has a very demanding control system with a pointing accuracy, the ACS design must be performed very carefully, since the flexible structure can become unstable when control loop is 

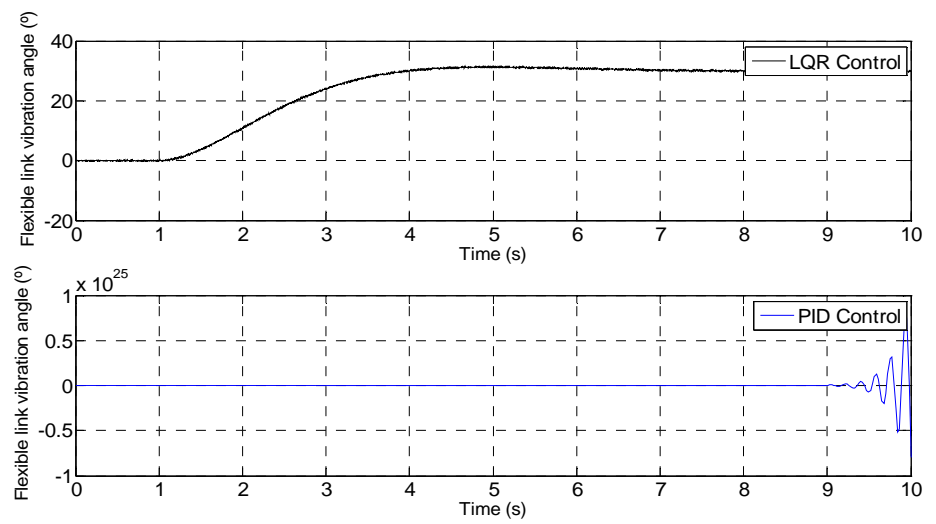

Fig. 8 PID and LQR, sensor at $x=0$.
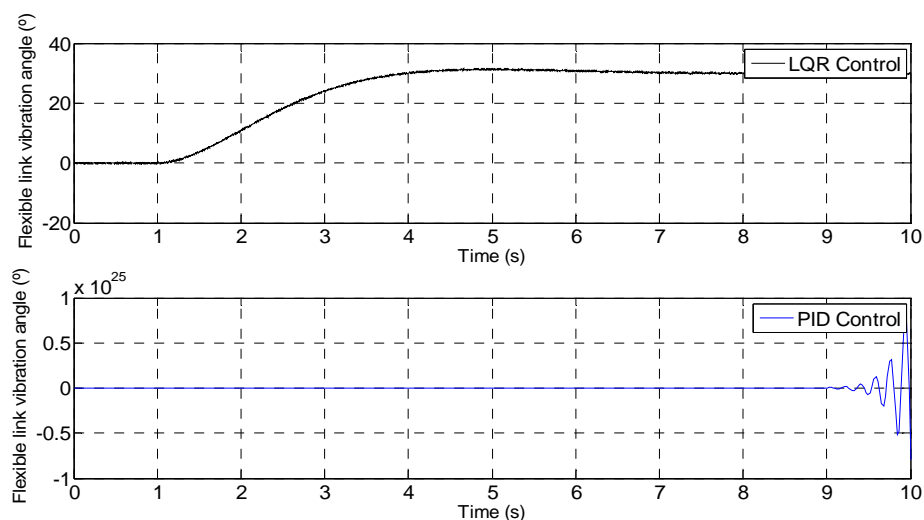

Fig. 9 PID and LQR, sensor at $x=0.788 \mathrm{~L}$.
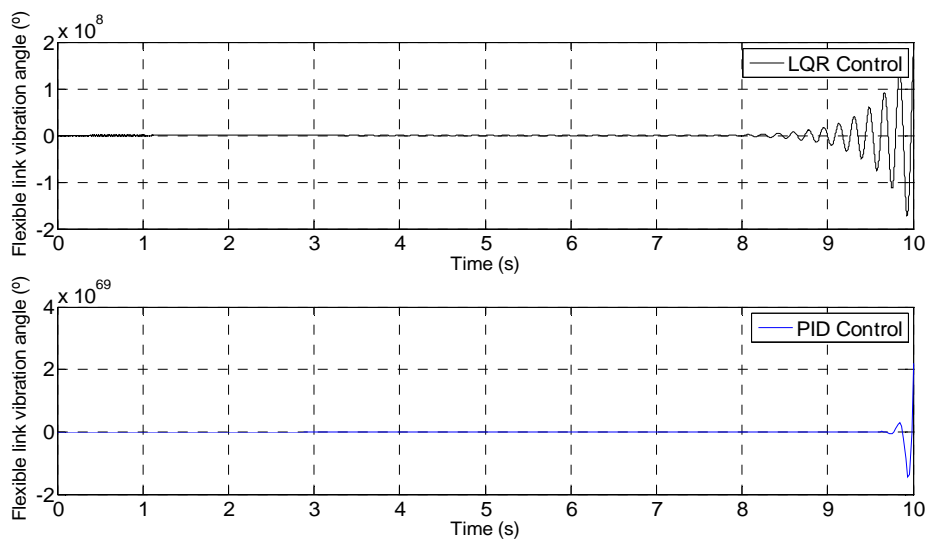

Fig. 10 PID and LQR, sensor at $x=L$.

feedback with the wrong information about the real attitude and flexible displacement of the satellite. It is important to know where is the exact point where the sensor/actuator problem starts to damage the dynamics stability of the system. For the specific system investigated here the transition point, where the zeroes cross the imaginary axis limit, is located at $78.88 \%$ of the structure length. Finally, one has to stress that the investigation here has considers only one flexible mode, and that an investigation with more modes is necessary.

\section{References}

[1] Spector, V. A., and Flashner, H. 1990. "Modeling and Design Implications of Noncollocated Control in Flexible Systems.” Journal of Dynamic Systems, Measurement and Control 112 (2): 186-93. 
[2] Park, J. H., and Asada, H. 1994. "Dynamic Analysis of Noncollocated Flexible Arms and Design of Torque Transmission Mechanisms." Journal of Dynamics Systems, Measurement and Control 116: 201-7.

[3] Wang, X. 2011. "Using LQG-LTR Control Law to Improve the Performance of Direct Drive Rotary Positioning System Subject to Uncertain Inertia Load.” In Proceedings of 2011 International Conference on Fluid Power and Mechatronics, 945-8.

[4] Sales, T. P., Rade, D. A., and Souza, L. C. G. 2013. "Passive Vibration Control of Flexible Spacecraft Using Shunted Piezoelectric Transducers.” Aerospace Science and Technology 1: 12-26.

[5] Pinheiro, E. R., Souza, and L. C. G. 2013. "Design of the Microsatellite Attitude Control System Using the Mixed Method via LMI Optimization.” Mathematical Problems in Engineering 2013 (1-8): 1-8.

[6] Souza, L. C. G., and Souza, A. G. 2014. "Satellite Attitude Control System Design Considering the Fuel Slosh Dynamics.” Shock and Vibration 1-8, 2014.

[7] Bigot, P., and Souza, L. C. 2014. "Investigation of the SDRE (State Dependent Riccati Equation) Adaptive
Control Advantages for Controlling Non-linear Systems as a Flexible Rotatory Beam.” International Journal of Systems Applications, Engineering and Development 8: 92-9.

[8] Conti G. T., and Souza, L. C. G. 2008. "Satellite Attitude Control System Simulator." Journal of Sound and Vibration 15 (3-4): 395-402.

[9] Gonzales, R. G., and Souza, L. C. G. 2009. “Application of the SDRE Method to Design a Control System Simulator." Advances in Astronautical Sciences 134: 2251-8.

[10] Rocha, T. L., Silva, S., and Junior, V. L. 2004. "Optimal Placement of Sensor and Actuator for Large Structures.” Presented at the 22nd IMAC Conference and Exposition: A Conference and Exposition on Structural Dynamics, Detroit, USA.

[11] Hale, J. M., and Daraji, A. H. 2012. "Optimal Placement of Sensors and Actuators for Active Vibration Reduction of a Flexible Structure Using a Genetic Algorithm Based on Modified H-infinity." J. Phys.: Conf. Ser. $\quad 382$ : 012036 doi:10.1088/1742-6596/382/1/012036. 